\title{
Enhance the Antimicrobial Activity of Silver Nanoparticles by Manipulating a Redox Process and Controlling the Size of the Particles
}

\author{
Nezar H Khdary ${ }^{1 *}$, Waleed K. Seddiq ${ }^{2}$, Maria E Alkurdi ${ }^{3}$, Essam J. Alyamani ${ }^{1}$ and Abdulaziz A ALangari ${ }^{4}$ \\ ${ }^{1}$ King Abdulaziz City for Science and Technology, Riyadh, Saudi Arabia \\ ${ }^{2}$ King Saud University, college of applied medical sciences, clinical laboratory sciences, Riyadh, Saudi Arabia \\ ${ }^{3}$ University of Massachusetts - Amherst, College of Natural Science, Biochemistry and Molecular Biology department, Massachusetts, USA \\ ${ }^{4}$ College of Medicine King Saud University, Riyadh, Saudi Arabia
}

*Corresponding author: Nezar H Khdary, King Abdulaziz City for Science and Technology, Riyadh, Saudi Arabia

\begin{abstract}
ARTICLE INFO
Received: 幽 March 25, 2019

Published: 幽 April 01, 2019

Citation: Nezar H Khdary, Waleed K. Seddiq, Maria E Alkurdi, Essam J. Alyamani, Abdulaziz A ALangari. Enhance the Antimicrobial Activity of Silver Nanoparticles by Manipulating a Redox Process and Controlling the Size of the Particles. Biomed J Sci \& Tech Res 16(4)-2019. BJSTR. MS.ID.002882.
\end{abstract}

Keywords: Silver Nanoparticles; $B a-$ copa; Green Synthesis; Antimicrobial Activity

\author{
ABSTRACT
}

Silver nanoparticles (Ag NPs) have many attractive properties such as electrical, thermal and optical properties comparing to other metal nanoparticles, consequently, there are many applications that depend on the Ag NPs particles, for example, optical, medical environmental, photovoltaic and, biological applications. This, therefore, leads to the importance of continuing to search for the best and least expensive methods of producing Ag NPs particles. In this study, Ag NPs were synthesized using a chemical redox process and a green process. In chemical redox process, three reducing agents have utilized sodium borohydride, hydrazine, and sodium citrate. In green synthesis the active compounds in Bacopa leaves were extracted using three different solvents; ultra-pure water, ethanol, and dichloromethane, then each extract was used and studied its effect on the formation of silver nanoparticles. Each method's influence on particle size was studied, as well as the potential of the produced Ag NPs to inhibit bacteria. The active compounds and Ag NPs were characterized using Fourier Transform InfraRed (IR) spectroscopy; UV spectroscopy. The particle morphology was imaged using FeldEmission Scanning Electron Microscopy. The particle-size distribution and zeta potential were measured using Malvern's Nano ZS 3600. This study produces three main results: First, certain synthesis parameters create specific particle sizes; second, Ag NPs' size significantly potential to inhibit bacteria; and third, the Ag NPs derived from the green redox method have more bacteria- inhibiting potential than the chemically synthesized ones have.

\section{Introduction}

In recent years, numerous scientific studies have investigated synthesized silver nanoparticles (Ag NPs) because of their beneficial properties, such as inhibiting bacterial growth [1]. In these studies, different methods of synthesis have been utilized, such as physical, photochemical, and biological methods [2]. The various methods produce Ag NPs with different shapes, sizes, and effectiveness of Ag NPs have shown varying potential to inhibit different types of bacteria [3]. Chemical and physical synthesis procedures often require hazardous chemicals and create toxic byproducts [4]. Therefore, many researchers have utilized green synthesis methods to overcome the toxicity problem and the requirement for hazardous chemicals5. Green methods (which are based on using green leaves) are eco-friendly and inexpensive, and they maintain energy. Furthermore, one study showed that using a Calliandra haematocephala leaf extract to synthesize Ag NPs created particle sizes that ranged from 13.54 to $91.28 \mathrm{~nm}$, which implies a polydisperse model6. Many studies have shown that Ag NPs are effective against pathogens such as bacteria, with some variations in effectiveness due to certain parameters. When some bacteria form a resistance to certain antibiotics, they may become a primary public-health problem [5-9]. Therefore, a new method and new materials to inhibit pathogenic bacteria must be developed. 
The Bacopa monnieri (L) plant, from the family Scrophulariaceae, is a perennial creeping plant found throughout many countries, including Saudi Arabia, often on the banks of valleys, on rivers, and in water [10]. The Bacopa leaf has many medical uses; for example, when turned into a drug, it can treat memory loss [11] and epilepsy [12]. A Bacopa leaf extract contains the chemical compound dammarane $\left(\mathrm{C}_{3} \mathrm{OH} 54\right)$, as well as other compounds that can reduce the Ag+ ions to Ag NPs because of the available functional groups of these compounds [13]. In this study, the Ag NPs are synthesized using a chemical redox process and a green process, and the two methods are then compared. A complete investigation identifies the reducing agents' effects not only on particle size but also on the ability of the produced Ag NPs to inhibit bacteria.

\section{Experimental Details}

\section{Materials}

Silver sulfate was obtained from Scharlau Chemie (Spain); ethanol, dichloromethane, and sodium borohydride were obtained from Prolabo (Europe); sodium citrate tribasic dihydrate and hydrazine were obtained from Sigma-Aldrich (United States). Water was purified using Integral water ultrapure purification system (Milli-Q Millipore, France). The Bacopa leaves were obtained from GPS location 24.3669720, 46.9072220, which is near Riyadh, Saudi Arabia.

\section{Extracting}

Several extraction methods were performed to identify the best extraction method for obtaining the active ingredients from Bacopa leaves. In the first method $\left(\mathrm{M}^{-1}\right)$, the Bacopa leaves were thoroughly washed with tap water, rinsed with deionized water several times to remove any soil particles, and dried in a desiccator under vacuum at $80^{\circ} \mathrm{C}$. Then the Bacopa leaves were weighed and transferred to a sterilized conical flask, to which $200 \mathrm{~mL}$ of deionized water was added, and the flask was left at $80^{\circ} \mathrm{C}$ for six hours. The yellow extract solution was filtered and transferred to a 200-mL evaporating dish. The dish was left over the warm-water bath until the solution concentrated to about $50 \mathrm{~mL}$. The second method (M-2) was the same as the previous method, except that $200 \mathrm{~mL}$ of ethanol was used instead of deionized water. To obtain all of the active-ingredient compounds, a third method (M-3) was performed at $\mathrm{pH}$ levels 4, 7, and 12 as follows: $60 \mathrm{~g}$ of washed and dried Bacopa leaves were mixed with $100 \mathrm{~mL}$ of ultrapure water, and the pHs were adjusted to the desired levels, with $0.1 \mathrm{M} \mathrm{HCl}$ for acidic media and $0.1 \mathrm{M} \mathrm{NaOH}$ for basic media. Each solution was then transferred to a separation funnel, $200 \mathrm{~mL}$ of dichloromethane was poured into each separation funnel, and each was shaken for 5 min and left for $10 \mathrm{~min}$. For each pH level, the organic layer (which contained the extracted compounds) was separated and placed in a porcelain evaporating dish and kept under vacuum at $25^{\circ} \mathrm{C}$. Last, all three $\mathrm{pH}$ portions $(4,7$, and 12$)$ were collected together.

\section{Green Synthesis of AgNPs}

The green synthesis of Ag NPs was performed by mixing a $90 \mathrm{mg}$ sample of the Bacopa extracts from all three methods (M ${ }^{1}, \mathrm{M}^{-2}$, and $\mathrm{M}^{-3}$ ) with $10 \mathrm{~mL}$ of silver nitrate solutions at different concentrations. The new batches were called $\mathrm{G}^{-1}, \mathrm{G}-2$, and $\mathrm{G}-3$, respectively. The reactions were allowed to continue until the suspension of silver nano-colloids was signified by a change in color from transparent to brown, which indicated the formation of Ag NPs.

\section{Chemical Synthesis of Ag NPs}

In order to investigate the effect of reducing and stabilizing agent on the size and distribution of the Ag NPs, redox chemistry was applied [13]. Three synthesis procedures were performed using three chemical agents: sodium borohydride $\left(\mathrm{NaBH}_{4}\right)$, hydrazine $\left(\mathrm{N}_{2} \mathrm{H}_{4}\right)$, and sodium citrate tribasic dihydrate $\left(\mathrm{C}_{6} \mathrm{H}_{5} \mathrm{Na}_{3} \mathrm{O}_{7} \cdot 2 \mathrm{H}_{2} \mathrm{O}\right)$. The reactions were performed as follows: $200 \mathrm{~mL}$ of $\mathrm{AgNO}_{3}(10$ $\mathrm{mM}$ ) was added to three flasks (A, B, and C). To Flask A, $70 \mathrm{~mL}$ of the $7 \mathrm{mM}$ of $\mathrm{NaBH}_{4}$ was added. To Flask B, $8.5 \mathrm{~mL}$ of $\mathrm{N}_{2} \mathrm{H}_{4}(80 \%$ in water) was added. To Flask C, $20 \mathrm{~mL}$ of $0.1 \mathrm{mM}$ of sodium citrate tribasic dihydrate was added. All flasks were left for 12 hours for the reaction to proceed at $25^{\circ} \mathrm{C}$ and continually received moderate stirring, and the colors of the three solutions changed gradually from transparent to dark brown, with slight differences in color intensity. The solutions were centrifuged at $9000 \mathrm{r} / \mathrm{min}$ for $30 \mathrm{~min}$, and the precipitates of the Ag NPs were taken and rinsed with deionized water and recovered by a centrifuge. The Ag NPs were dispersed in $10 \mathrm{~mL}$ of deionized water to be prepared for characterizations and the effectiveness test.

\section{Antimicrobial Activity}

The antimicrobial activity of the Ag NPs was evaluated against Escherichia coli (ATCC 25922) and Staphylococcus aureus (ATCC 25923) with the disc diffusion method [14]. All experimental components were sterilized at $110^{\circ} \mathrm{C}$ overnight. The cultures aged $24 \mathrm{~h}$ were derived from a stock of Mueller Hinton broth. The bacteria were transferred to the cultures through spreader holes placed on agar plates, and a $100 \mu \mathrm{L}$ of stabilized synthesized Ag NPs was placed on the spreader holes. The plates were incubated at $37^{\circ} \mathrm{C}$ overnight.

\section{Characterizations}

The morphology of the Ag NPs was studied with a field emission scanning electron microscopy (FESEM) (JEOL, JSM-7800F), and the optical images were taken using the Motic BA310 compound microscope. The size distribution and the zeta potential were measured with Malvern's Nano ZS 3600. Absorbencies of the active ingredients and their complexes with the Ag NPs were represented using the Shimadzu UV-3600 spectrophotometer. For absorbance measurements, a $2 \mathrm{~mL}$ solution was transferred to a $3 \mathrm{~mL} \mathrm{UV-Vis}$ quartz cuvette, and the measurement of absorbance used the range 200-500 $\mathrm{nm}$. The infrared spectra were evaluated under vacuum with Bruker's VERTEX 70v spectrophotometer and a resolution of $4 \mathrm{~cm}^{-1}$, and 64 scans were taken. Before the measurements, drops of a solution were placed onto an attenuated total reflection (ATR) attachment. The ATR sampling aided the direct investigation of the unprepared liquid sample, and the effects of atmospheric water and $\mathrm{CO}_{2}$ were eliminated by using an ultra-vacuum pump integrated within the system. 


\section{Results and Discussion}

The particle sizes obtained from the chemical synthesis procedures (in which sodium citrate tribasic dihydrate, sodium borohydride, and hydrazine were used) are illustrated in Schematic 1 . Schematic 2 shows the results of the green synthesis procedures, in which the Bacopa extract was prepared in three media. Both schematic diagrams clearly show that the reducing agents in both types of procedures altered particle size. For example, smaller particle size was achieved with sodium citrate tribasic dihydrate (53 $\pm 16 \mathrm{~nm}$ ), as shown in Figure 1a. When hydrazine was used, the particles became more prominent and a polydisperse model was observed, as shown in Figure 1c. $\mathrm{NaBH}_{4}$ produced particle sizes around $136 \pm 26 \mathrm{~nm}$, as shown in Figure 1b. sodium citrate tribasic dihydrate produced the best results, which can be attributed to the different factors that can affect the reaction rate $[15,16]$. Hydrazine produced a slow reaction rate, $\mathrm{NaBH}_{4}$ produced a fast rate, and sodium citrate produced a rate that was between those of hydrazine and $\mathrm{NaBH}_{4}$. Therefore, controlling the speed of the reduction reaction enables the control of the particle size and improves stability. The stability of Ag NPs is attributed to the negative charge of an associated ion such as borohydride ion which distributed on the surface of nanoparticles leading to avoiding the aggregation of particles due to the electrostatic repulsion13. Zeta potential measurements showed that G1, G2, and G3 have $-28,-22$ and $-40.5 \mathrm{mV}$ respectively.

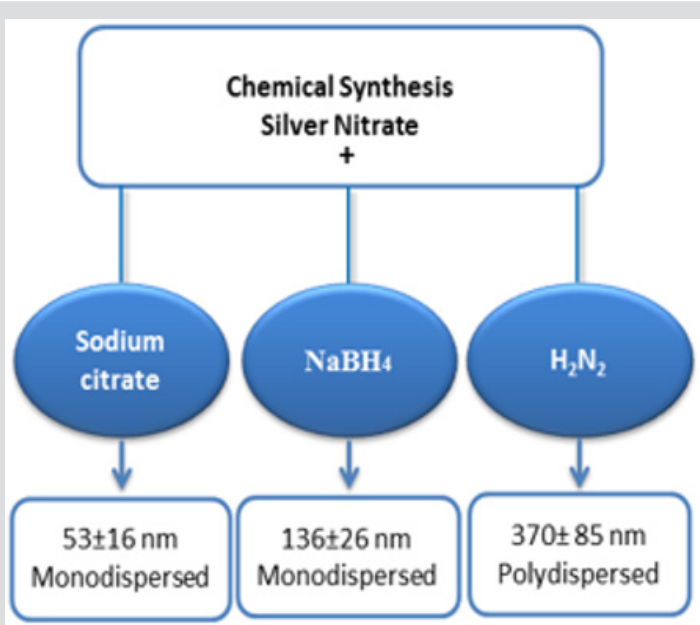

Schematic 1: The effect of the chemical reducing agent on the size of AgNPs.

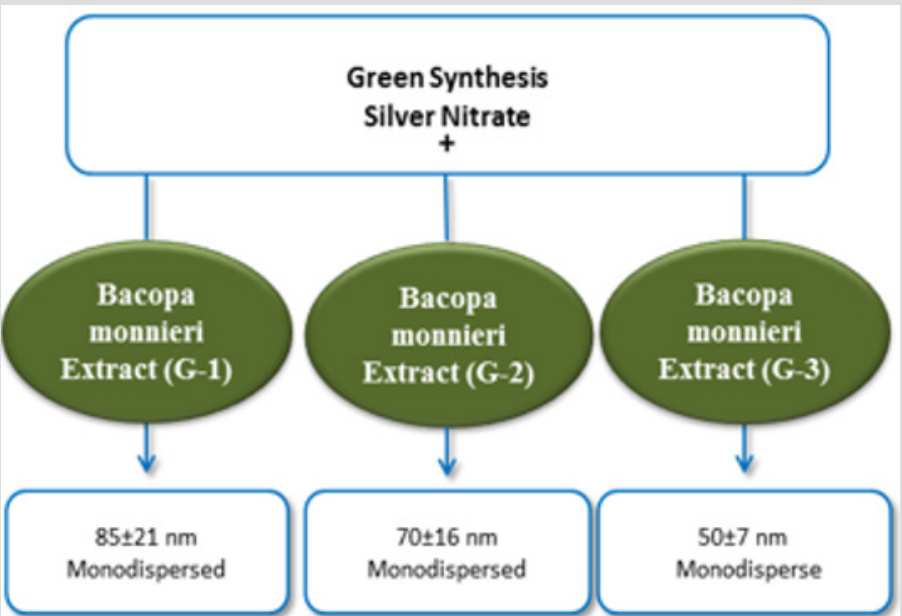

Schematic 2: The effect of green reducing agent on the size of AgNPs.

This means that the best stable particles in the solution are the resulting from synthesis G-2 which consistent with the value of zeta potential for nanoparticles stability in a solution which is lies between $>+30$ and $<-30 \mathrm{Mv}[17,18]$. The green synthesis methods produced better results, as illustrated in Schematic 2. For example, all of the green synthesis methods produced a monodisperse model, and the sizes of the particles were less than $100 \mathrm{~nm}$. Figure $1 \mathrm{~d}$ shows the particle-size distribution of Ag NPs synthesized with the G-3 method. (Figures 1d,1e \& 1f) show that Bacopa extracts obtained at different $\mathrm{pH}$ levels produced suitable particle sizes. This result can be attributed to some of Bacopa monnieri's chemical compounds such as bacopaside, bacosterol. These compounds work as antioxidants, contribute to the reduction process, and maintain a uniform particle size [17]. Therefore, the sodium citrate in the chemical synthesis procedure generated the best results among other reducing agents. Moreover, the Bacopa extract used in the G-3 method produced better results than the M-3 method, in which Bacopa extracts dependent on $\mathrm{pH}$ levels were used. It is clear that the use of green synthesis methods does not cause any obstacle, where the active materials are extracted using an appropriate solvent and then used to reduce Ag+ to Ag metal nanoparticles [14]. 


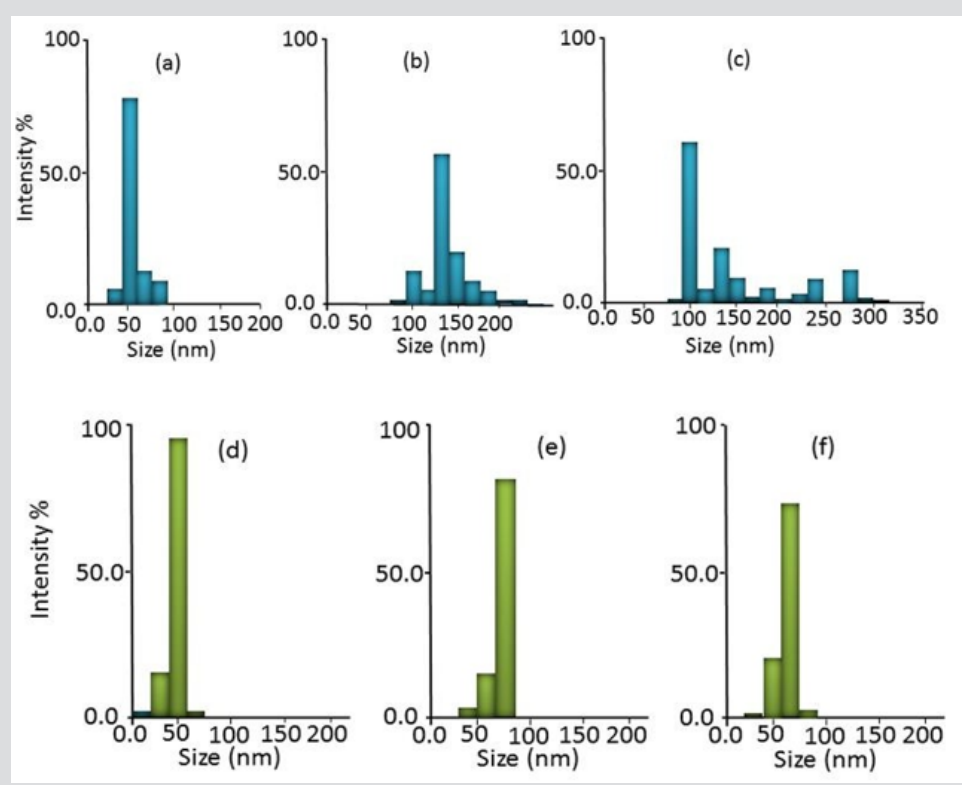

Figure 1: Histograms showing the particle size distribution of Ag NPs produced with (a) sodium citrate, (b) sodium borohydrid, and (c) hydrazine, as well as Ag NPs produced with Bacopa extracts made with (d) DMC, (e) ethanol, and (f) water.

Figure 2 shows the effect of the Ag NP concentration on the color intensity of the produced particles, and the changes in color are due to the intensity of Ag NPs and the size of the particles. To investigate the effect of the extract composition on the color intensity, two batches were selected one from G-2 and the other from G-3. Figure 2 shows that each extract produced different colors intensity, despite the concentrations of silver never being changed. These results confirm that the compounds in the Bacopa extract using ethanol (G-2) and in the extract using different $\mathrm{pH}$ levels (G-3) produce different color intensities, which can be attributed to the nature of the extracts because the solvent used and the $\mathrm{pH}$ levels play important roles in extracting specific compounds from the substance $[19,20]$. The Bacopa extracts were investigated under a polarized light microscope. Figure 3a shows the crystals before their incorporation with Ag NPs, and the crystals have a needlelike shape. The color intensity depends on the crystals' thickness and distribution on the slide, as shown in (Figure 2a). However, when the crystals incorporated with Ag+ ions and were used as a reducing agent, an amorphous new crystal structure formed, as shown in Figure 3b. The destruction or aggregation of the large crystal can account for this phenomenon [21].

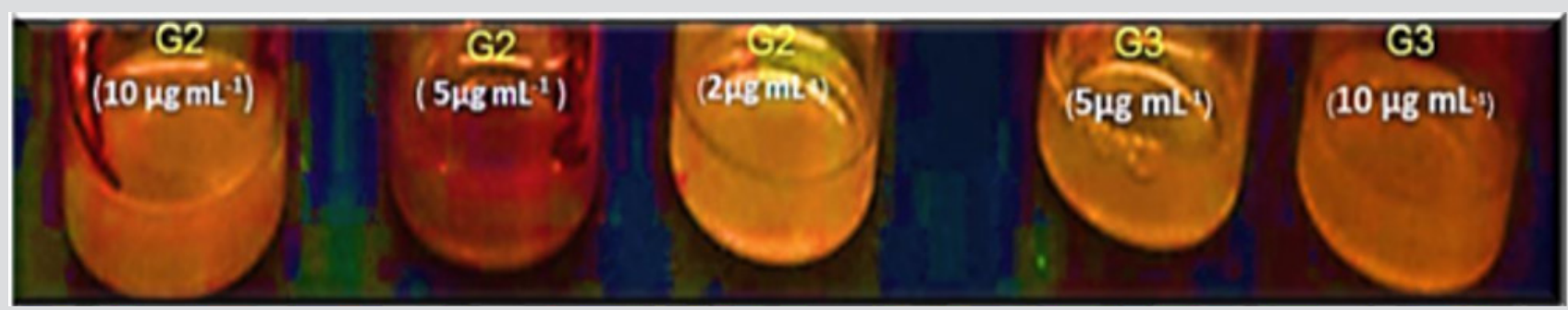

Figure 2: Change in color due to a variation of silver concentration.

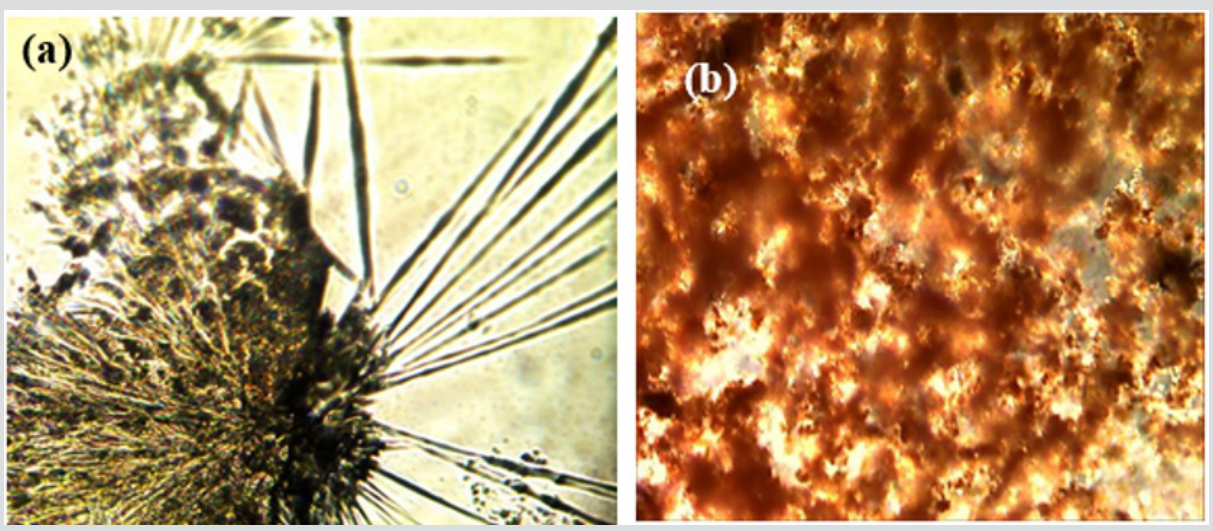

Figure 3: (a) Optical microscopic image of the Bacopa extract after crystallization and (b) same crystals after adding Ag solution. 
The morphologies of the Ag NPs produced in the chemical and the green synthesis procedures are shown in Figure 4. Figures $4 \mathrm{a} \& 4 \mathrm{c}$ show that the shape of the particles was spherical with some aggregation. At high magnification, the spherical structure is apparent, and the particle sizes are less than $100 \mathrm{~nm}$. Figure 5 shows the Infrared spectrum plot of wavenumber vs \% transmittance of the Bacopa monnieri pH-dependent extracts with $\mathrm{CH} 2 \mathrm{Cl} 2$ (Figure $5 a)$ and with water (Figure 5a). Numbers of bands were clearly observed. These bands are associated with the structures of Bacopa extracted compounds. For example, one of these compounds was bacoside (Figure 6). (Figure 5a) shows the IR spectra of the pHdependent extracts. A number of bands are ascribed to the presence of chemical bonds and functional groups. The band at $704 \mathrm{~cm}^{-1}$ attributed to $\mathrm{C}=\mathrm{C}$ bending alkene and the band at $1039 \mathrm{~cm}^{-1}$ is due to the C-O stretching where the band at $1047 \mathrm{~cm}^{-1}$ corresponded to $\mathrm{C}-\mathrm{O}$ in primary alcohol. The band at $1124 \mathrm{~cm}^{-1}$ is associated with C-O stretching aliphatic and the band at $1287 \mathrm{~cm}^{-1}$ assigned to C-O stretching ether. The band at $1381 \mathrm{~cm}^{-1}$ is accredited to $\mathrm{OH}$ bending. At $1463 \mathrm{~cm}^{-1}$ the $\mathrm{C}-\mathrm{H}$ is clearly observed due to bending of a methylene group. The band at $1727 \mathrm{~cm}^{-1}$ ascribed to $\mathrm{C}=0$ stretching aliphatic ketone. The band at 2872 and $2928 \mathrm{~cm}^{-1}$ attributed to the stretching of C-H. The band at 2959 and 3436 are assigned to the stretching alcohol22.
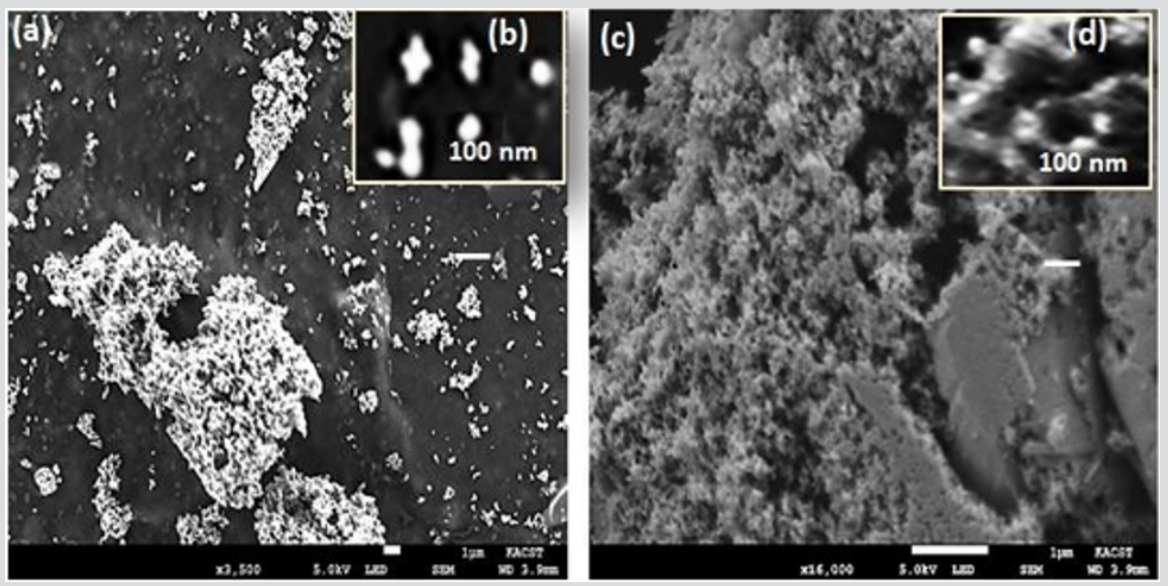

Figure 4: FE-SEM images of (a) Ag NPs synthesis by chemical process using sodium citrate (M-3) and (b) at high magnification; and (c) Ag NPs synthesized using Bacopa extract (G-3) synthesis and (d) at high magnification.

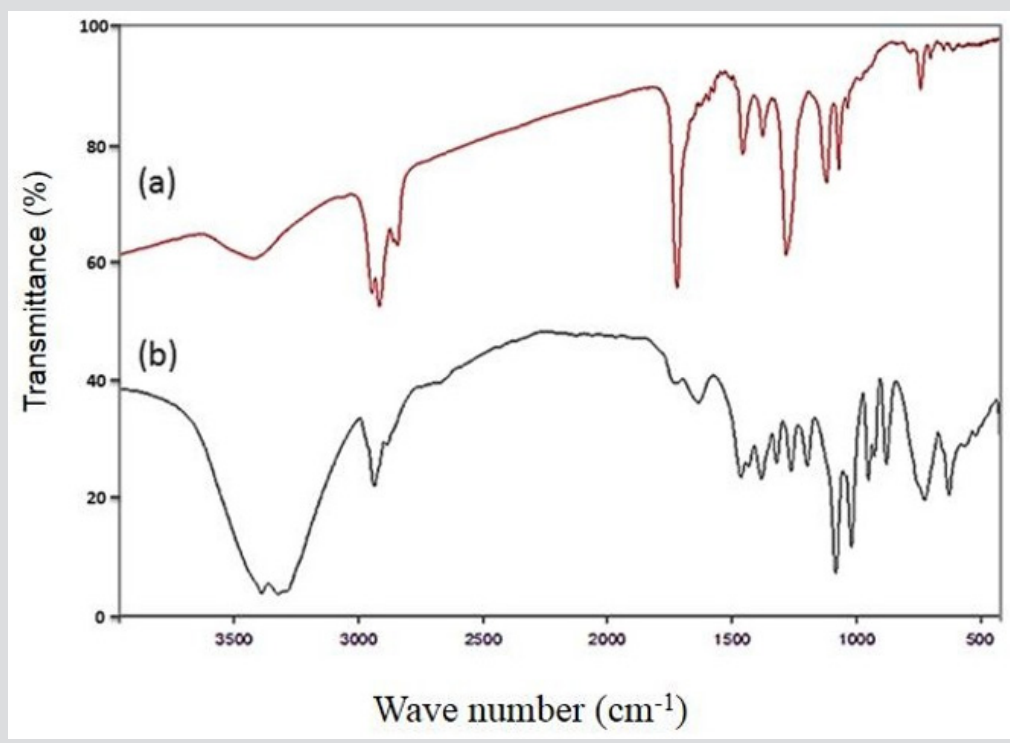

Figure 5: FTIR of (a) pH-dependent extract and (b) ethanolic extract.

A spectrum (b) for water-based extraction shows peaks at 1019 and $1082 \mathrm{~cm}^{-1}$ attributed to $\mathrm{C}$ - 0 stretching. The band at $1727 \mathrm{~cm}^{-1}$ is associated with carbonyl group located in cyclopentane ring in the bacoside molecule. The band at $1463 \mathrm{~cm}^{-1}$ is associated with C-C stretching vibration and band at $1635 \mathrm{~cm}^{-1}$ is consequent of stretching vibration of $\mathrm{C}=\mathrm{C}$ [22]. The 627,725 and 878, 927, 951 $\mathrm{cm}^{-1}$ ascribed to the $\mathrm{C}-\mathrm{H}$ and $=\mathrm{C}-\mathrm{H}$ bending. The bands at 1082, 1197 and $1320 \mathrm{~cm}^{-1}$ attributed to C-O stretching. The band 2965 and $1380 \mathrm{~cm}^{-1}$ ascribed to $\mathrm{C}-\mathrm{H}$ bending. The band at 3322 and 3388 $\mathrm{cm}^{-1}$ is ascribed to $\mathrm{O}-\mathrm{H}$ stretching. The band at $627 \mathrm{~cm}^{-1}$ is attributed to sp2 $\mathrm{C}-\mathrm{H}$ stretching, and the band $1019 \mathrm{~cm}^{-1}$ is attributed to $\mathrm{C}-\mathrm{O}$ in $\mathrm{C}-\mathrm{O}-\mathrm{H}$, as well as the band at $3322 \mathrm{~cm}^{-1}$. All of them are related to the Bacopa extracts. These results confirm the successfully extracted of the Bacopa active ingredients. Also the spectra (a) pH-dependent extracts showed a redshift, which could be due to hydrogen bonds [23]. These results also confirm that the Bacopa leaves obtained 
from Riyadh, Saudi Arabia, contain all of the compounds indicated in Bacopa extracts from India [21]. This confirms that the original environment did not significantly affect the active substance. Figure
7 shows the UV-Vis absorbance of the Bacopa extract in ethanol (a) and dichloromethane (DMC) (b).

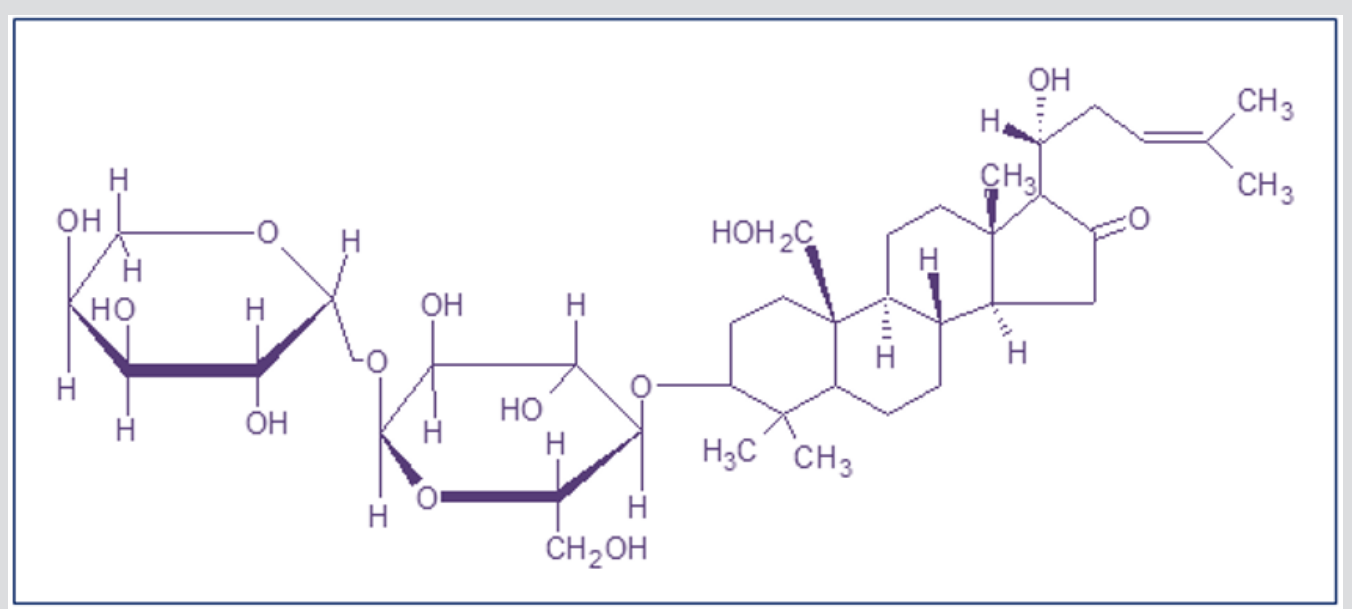

Figure 6: Structure of bacoside-A.
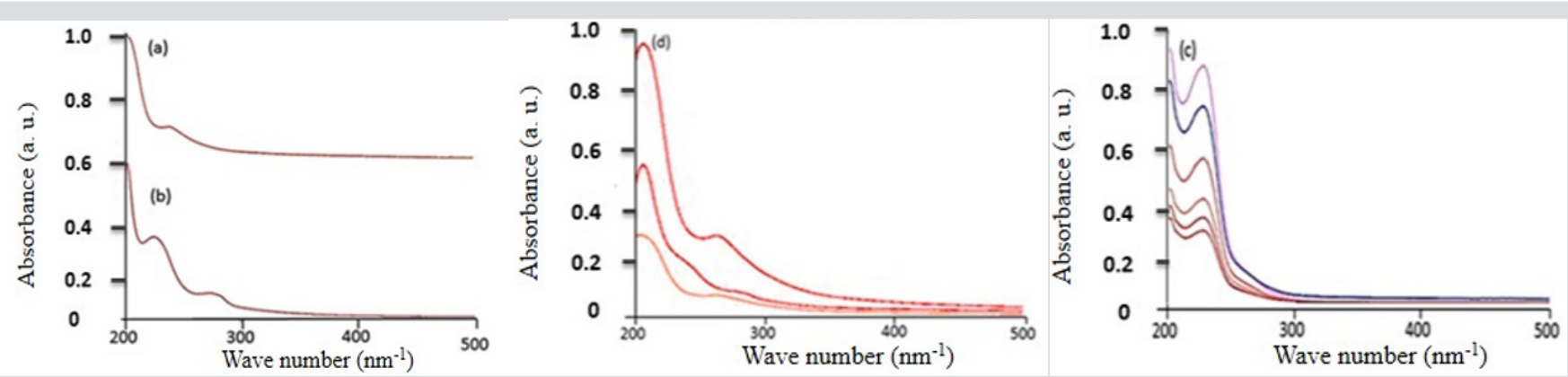

Figure 7: UV-Vis of the Bacopa extract using (a) ethanol, (b) using DMC at different $\mathrm{pH}$, (c) different concentrations of the Bacopa extract, and (d) using various amounts of Bacopa extract (2.0, 4.0, $8.0 \mathrm{ml}$ ) mixed with Ag+ to complete $10 \mathrm{ml}$ Ag NPs solution.

The extractions that used different $\mathrm{pH}$ levels and DMC are superior when compared with the ethanol-based extract. The absorption peak at $217 \mathrm{~nm}$ can be attributed to the electron transition from $\pi-\pi^{*}$, also known as the HOMO LUMO gap. This confirms the presence of the pi bonds, which are shown in the structure of bacoside-A. Moreover, in the DMC extract, another peak appears around $278 \mathrm{~nm}$, indicating that the method of extract collection at acid, natural, and basic pH levels with DMC is better than the ethanol- or water-based methods because the DMC method can obtain more compounds. To confirm the association between the concentrations and the absorption of the extracted materials, different concentrations were measured. Figure $7 \mathrm{c}$ shows a direct correlation between the concentrations and the absorption intensities, and the correlation is due to the increased concentrations of the Bacopa compounds. Figure $7 \mathrm{c}$ shows the spectra of the Ag NPs when mixed with 2.0, 5.0, and $8.0 \mathrm{~mL}$ Bacopa extracts. At the first and second spectra with 2.0 and 5.0 $\mathrm{mL}$ concentrations, the peak at $278 \mathrm{~nm}$ almost disappeared. This disappearance can be attributed to the Bacopa extracts' active ingredients being used to reduce the Ag+ ions to Ag NPs. The peak at $278 \mathrm{~nm}$ appeared in the $8.0 \mathrm{~mL}$ concentration implying not only that at some level, all of the Ag+ ions converted to Ag NPs but also that no more Ag+ ions were in the solution [24]. Therefore, the rest of the Bacopa extracts will start to rise at $278 \mathrm{~nm}$. In addition, (Figure $7 \mathrm{~d}$ ) shows a redshift on the spectra around $5 \mathrm{~nm}$ to a more extended wavelength; this may be due to the surface plasmonic effect spouting from the formation of the Ag NPs [25].

\section{Antimicrobial Activity}

The disc diffusion method was used to evaluate the antimicrobial activity of Ag NPs (those created with and without Bacopa extracts) against Staphylococcus aureus and Escherichia coli [26]. Figures 8a \& 8b show that Ag NPs synthesized with a chemical reduction process slightly affect Staphylococcus aureus and Escherichia coli except for the Ag NPs synthesized with sodium citrate. These Ag NPs produced inhibition zones of $12 \mathrm{~mm}$ for Escherichia coli and $10 \mathrm{~mm}$ for Staphylococcus aureus, and these results may be due to the effect of the reaction rate, which was calculated with Equation 1. The reaction rate was in this order: sodium borohydride > hydrazine > sodium citrate tribasic dihydrate. Therefore, the reaction rate plays a key role in determining particle size, and that role is reflected in the effectiveness of the produced Ag NPs to inhibit the bacteria. This result is confirmed by the particles sizes obtained with different reducing agents. Sodium citrate tribasic dihydrate produced particle sizes around $5 \mathrm{~nm}$, and these $\mathrm{Ag}$ NPs had a monodisperse model because this reaction used more 
time to form Ag NPs than did sodium borohydride and hydrazine. Therefore, the Ag NPs produced by sodium citrate tribasic dihydrate have a greater capability to inhibit bacteria, and the particles' size and monodisperse model can account for this capability which is consistent with the conclusion stated by Raj et al. [27].
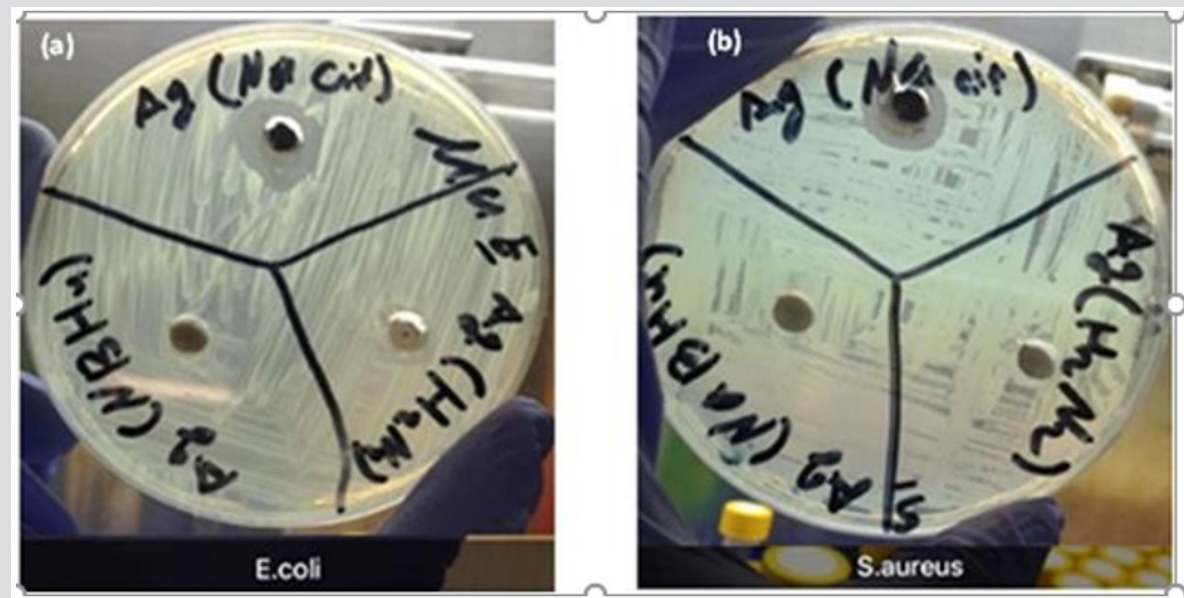

Figure 8a b: a. Inhibition zone at the dish plate Escherichia coli (bacteria) for the chemical synthesis. b)Inhibition zone at the dish plate Staphylococcus aureus (bacteria) for the chemical synthesis.

Figures 8c \& 8d show the effects of Ag NPs produced with the green synthesis procedures, which created Ag NPs that can inhibit bacterial growth more effectively than those created by chemical syntheses. Furthermore, the Ag NPs produced in G-3 exhibited the most antimicrobial activity: The inhibition zones were $17 \mathrm{~mm}$ for Escherichia coli and $13.8 \mathrm{~mm}$ for Staphylococcus aureus, with the concentration of $15 \mu \mathrm{g} \mathrm{mL}^{-1}$. These levels of inhibition are preferable than the one obtained by Paredes et al. [25]. The G-2 batch produced smaller inhibition zones: $15 \mathrm{~mm}$ for Escherichia coli and $11 \mathrm{~mm}$ for Staphylococcus aureus. Two variables can be offered to account for this result: the particle sizes and the particles' distribution models [28]. As Figures 1d,1e, \& 1f show, the particle sizes obtained with green syntheses were around $50 \mathrm{~nm}$, and the dispersion models closely resembled a mono form. To study the inhibitory effects of the Ag NPs and Ag+ ions on Escherichia coli and Staphylococcus aureus, fractions obtained from the green syntheses were implemented. Table 1 and 2 show the concentrations' effects on the inhibition of Escherichia coli and Staphylococcus aureus. The Ag NPs inhibited both types of bacteria, but they impeded Escherichia coli more than Staphylococcus aureus
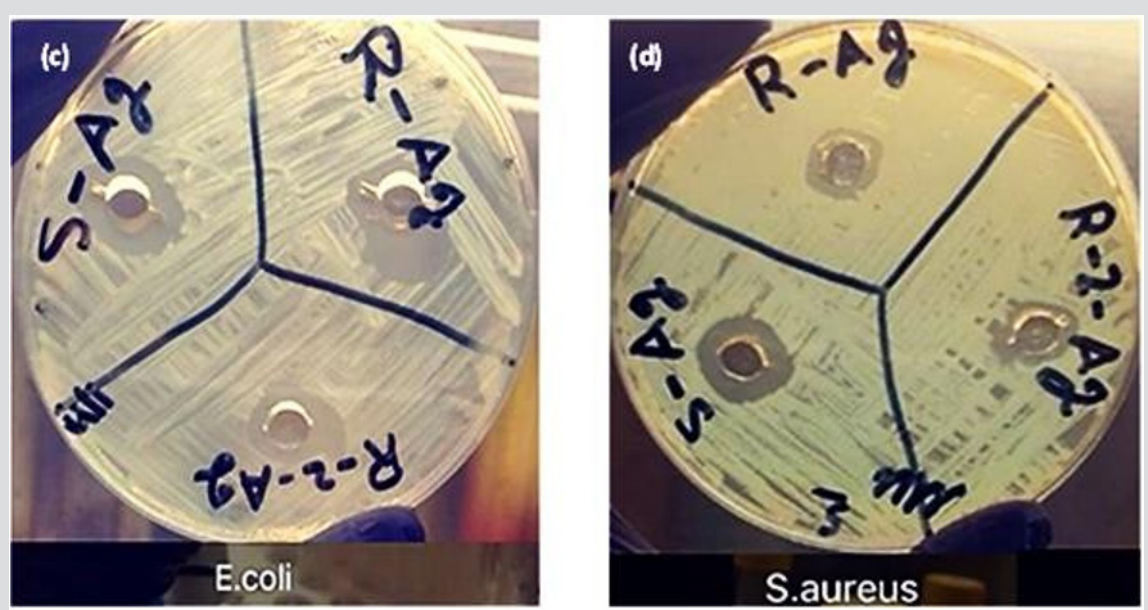

Figure 8c d: c. The activity of silver nanoparticles versus Escherichia Coli (G-3), (G-2), and(G-1). d: Activity of silver nanoparticles versus Staphylococcus aureus (G-3), G-2) and (G-1).

Table 1: The effect of AgNPs (G-1, G-2, and G-3) on Escherichia coli.

\begin{tabular}{|c|c|c|c|c|}
\hline \multirow{2}{*}{ Concentration $\mathbf{u g ~ m L}^{-\mathbf{1}}$} & \multicolumn{3}{|c|}{ Inhibition zone (mm) in Escherichia coli } \\
\cline { 2 - 5 } & G-3 & G-2 & G-1 & 10 \\
\hline 2 & 15 & 13 & 11 & 11 \\
\hline 5 & 16 & 14 & 13 & 13 \\
\hline 10 & 17 & 15 & $\mathbf{g}^{+}$ \\
\hline
\end{tabular}


Table 2: The effect AgNPs (G-1, G-2, and G3) on Staphylococcus aureus.

\begin{tabular}{|c|c|c|c|c|}
\hline \multirow{2}{*}{ Concentration $\boldsymbol{\mu g} \mathbf{~ m L}^{-\mathbf{1}}$} & \multicolumn{4}{|c|}{ Inhibition zone (mm) in Staphylococcus aureus } \\
\cline { 2 - 5 } & $\mathbf{G - 3}$ & $\mathbf{G - 2}$ & $\mathbf{G}-\mathbf{1}$ & $\mathbf{A g}^{+}$ \\
\hline 2 & 12 & 10 & 10 & 12 \\
\hline 5 & 13 & 21 & 11 & 13.7 \\
\hline 10 & 14 & 12 & 12 & 14 \\
\hline
\end{tabular}

\section{Conclusion}

In this study, two methods were used to synthesize Ag NPs: chemical reducing agents and Bacopa extracts. The using of chemical reducing agent for sodium borohydride, hydrazine and sodium citrate showed the marked difference as the size obtained 53, 136 and $370 \mathrm{~nm}$ in diameter respectively. Whereas, the synthesis using green syntheses showed a slight change in sizes and all syntheses products were less than $100 \mathrm{~nm}$. The Ag NP synthesized by green method demonstrated high inhibition of bacteria. The green syntheses of Ag NPs obtained better particle sizes and distribution model and exhibited more antibacterial activity. This study's results should encourage and inform the development of new applications that employ this study's extraction method, which can produce highly efficient nanoparticles for medical purposes.

\section{Acknowledgment}

The authors thank and appreciate the King Abdulaziz City of Science and Technology (KACST) for their technical support. Also, the authors thank the Deanship of Scientific research, College of Applied Medical Science Research Center at King Saud University.

\section{References}

1. J Natsuki, T Natsuki, Y Hashimoto (2015) A Review of Silver Nanoparticles: Synthesis Methods, Properties and Applications. Int J Mater Sci and Applic 4(5): 325-332.

2. J Kashmira, A Patel (2010) A review on Bacopa monniera: Current research and future prospects. Int J Green Pharm, pp. 156.

3. T Theivasanthi, M Alagar (2011) Anti-bacterial Studies of Silver Nanoparticles, pp. 1101.

4. A Gliga, S Skoglund, I Wallinder, H Karlsson (2014) Size-dependent cytotoxicity of silver nanoparticles in human lung cells: the role of cellular uptake, agglomeration and Ag release. Particle and Fibre Toxicology 17: 11 .

5. B Mahitha, B Raju, G Dillip, C Reddy, K Mallikarjuna, et al. (2015) Biosynthesis, characterization and antimicrobial studies of AgNPs extract from Bacopa monniera whole plant. Dig J Nanomater Biostruct 6(1): 135-142.

6. S Raja, V Ramesh, V Thivaharan (2015) Green synthesis, structural characterization, and catalytic activity of silver nanoparticles stabilized with Bridelia retusa leaf extract. J Saudi Chem Soc.

7. L Wang, C Hu, L Shao (2017) The antimicrobial activity of nanoparticles: present situation and prospects for the future. Int J NanoMed 12: 12271249.

8. W Salema, D Leitnera, F Zingla, G Schratterc, R Prassl, et al. (2015) Essential Oils and Nanotechnology for Treatment of Microbial Diseases. Int J Med Microbiol, pp. 305.

9. M Kumar A Curtis, C Hoskins (2018) Application of Nanoparticle Technologies in the Combat against Anti-Microbial Resistance. Pharmaceutics 10(1): 11.

10. J Kean, L Downey, C Stough (2017) Systematic Overview of Bacopa monnieri (L.) Wettst. Dominant Poly-Herbal Formulas in Children and Adolescents. Med Basel Switzerland 4(4): 86.
11. S Roodenrys, D Booth, S Bulzomi, A Phipps, C Micallef, et al. (2002) Chronic effects of Brahmi (Bacopa monnieri) on human memory. Neuropsychopharmacology 27(2): 279-281.

12. S Aguiar, T Borowski (2013) Neuropharmacological Review of the Nootropic Herb Bacopa monnieri. Rejuvenation Res 16(4): 313-326.

13. L Mulfinger, SD Solomon, M Bahadory, A Jeyarajasingam, S Rutkowsky, et al. (2007) Synthesis and Study of Silver Nanoparticles. J Chem Educ 84(2): 322 .

14. M Serrano, M Serrano, M Ramírez, D Morilla, A Valverde, et al. (2004) Evaluation of Disk Diffusion Method for Determining Posaconazole Susceptibility of Filamentous Fungi: Comparison with CLSI Broth Microdilution Method. J Antimicrob Chemother 50(3): 1108-1111.

15. Y Zhang, M Yang, NG Portney, D Cui, G Budak, et al. (2008) A SurfaceCharge Study on Cellular-Uptake Behavior of F3-Peptide-Conjugated Iron Oxide Nanoparticles. Biomed Microdevices 5(17): 10.

16. J Turkevich, P Stevenson, J Hillier (1951) A Dual Effect of AuNanoparticles on Nucleic Acid Cholesteric Liquid-Crystalline Particles. Discussions of the Faraday Soc, p. 11.

17. N Thanh, N Maclean, S Mahiddine (2014) Mechanisms of Nucleation and Growth of Nanoparticles in Solution. Chem Rev 114 (15): 7610-7630.

18. V Makarov, A Love, O Sinitsyna, S Makarova, I Yaminsky, et al. (2014) "Green" Nanotechnologies: Synthesis of Metal Nanoparticles Using Plants. Acta Naturae 6(1): 35-44.

19. K Gohil, J Pate (2010) A review on Bacopa monniera: Current research and future prospects. Int J Green Pharm, p. 4.

20. A Al-snafi (2013) The Chemical Constituents and Pharmacological Importance of Celosia Cristata-A Review. Int J Pharma Sci Res 5(4): 254 261.

21. Q Do, A Angkawijaya, P Tran Nguyen, L Huynh, F Soetaredjo, et al. (2014) Effect of extraction solvent on total phenol content, total flavonoid content, and antioxidant activity of Limnophila aromatica. J Food Drug Anal, pp. 296.

22. J Coates (2000) Encyclopedia of Analytical Chemistry R.A. Meyers (Edn.). John Wiley \& Sons Ltd Chichester, pp.10815.

23. J Joseph, E Jemmis (2007) A Unifying Electron-Counting Rule for Macropolyhedral Boranes, Metallaboranes, and Metallocenes. J AM Chem Soc p. 21.

24. X Zhang, Z G Liu, W Shen (2016) Silver Nanoparticles: Synthesis, Characterization, Properties, Applications, and Therapeutic Approaches. Int J Mol Sci 17(9): 1534.

25. X Fan, W Zheng, D Singh (2014) Light scattering and surface plasmons on small spherical particles. J Light Sci Appl, pp. 179.

26. M Lehtopolku, P Kotilainen, P Puukka, U Nakari, A Siitonen, et al. (2012) Prevalence and Antimicrobial Resistance of Campylobacter Isolated from Dressed Beef Carcasses and Raw Milk in Tanzania. J Clin Microbiol 22(1): 40-52.

27. A Raj, P Shah, N Agrawal (2017) Dose-dependent effect of silver nanoparticles (AgNPs) on fertility and survival of Drosophila: An in-vivo study. PLOS ONE 178051.

28. D Paredes, C Ortiz, R Torres (2014) Synthesis, characterization, and evaluation of antibacterial effect of Ag nanoparticles against Escherichia coli 0157:H7 and methicillin-resistant Staphylococcus aureus (MRSA). Int J NanoMed 9: 1717-1729. 


\section{ISSN: 2574-1241}

DOI: 10.26717/BJSTR.2019.16.002882

Nezar H Khdary. Biomed J Sci \& Tech Res

(C) (P) This work is licensed under Creative

Submission Link: https://biomedres.us/submit-manuscript.php

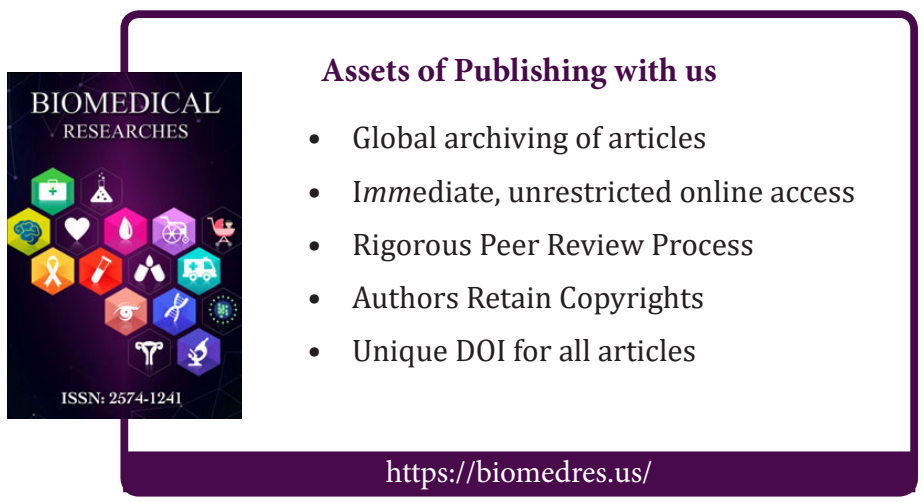

